\title{
IMPACT DE L'EXPLOITATION MINIERE SUR LES INFRASTRUCTURES ET LA VIE SOCIALE DANS LA VILLE DE KOLWEZI
}

\author{
Par Antoine NGOY, Papy MWAND, Giscard BAHALA KOBA et Elie KAHILU \\ MUKAZU
}

Dans un Etat de Droit, soucieux des sauvegarder les droits fondamentaux de la liberté de sa population et de veiller sur la vie sociale de cette population, l'administration évite de se réserver de trop large aux entreprises qui ne remplissent pas leurs devoirs pour le bien être de la communauté.

En effet, notre étude porte sur l'impact de l'exploitation minière sur les infrastructures et la vie sociale dans la ville de Kolwezi. Il convient dès à présent, que nous définissions les concepts impacts, exploitation minière, les infrastructures ainsi que la vie sociale.

L'existence des entreprises minières dans une ville est un atout, un avantage primaire pour la population de cette entité, dès lors qu'il y a création des emplois par le gouvernement (lutte contre le chômage), contribution pour la construction de routes, amélioration des conditions de vie de la communauté, la construction des ponts, des aéroports modernes, d'une gare, les construction des écoles, des universités ou des centres des recherches, l'aménagement des jardins botaniques ou zoologiques, l'aménagement d'un trottoir et la construction d'un centre hospitalier, d'un marché public, l'aménagement des terrains des sports et des lieux de loisir, etc.

Ainsi donc, l'existence des entreprises minières dans une entité devient un atout dans la vie sociale de la population. Ces quelques exemples cités ci-haut, illustre les cas le plus divers ou l'intérêt public peut se manifester et où l'administration peut valablement intervenir si les devoirs et obligations ne sont pas remplis.

Jean RIVERO et Jean WALINE renseignent que le but de l'administration, c'est la poursuite de l'intérêt public, le domaine de l'administration est constitué des nécessités auxquelles l'initiative privée ne peut répondre et sont vitales pour la communauté entière et pour chacun de ses membres.

C'est la sphère de l'intérêt public.

Le moteur de l'action administrative est la poursuite de l'intérêt général, ou encore de l'utilité publique, ou dans une perspective plus philosophique du bien commun. L'intérêt général est un ensemble de nécessités humaines. Ainsi, l'exploitation minière serait pour la communauté un intérêt général dans le sens où elle améliore les conditions de vie de l'entité. 
Dans le cadre de nos recherches, la problématique désignerait l'ensemble d'idées qui signifieront la position du problème suscité par le sujet d'étude. Ainsi, en ce qui concerne le présent travail, les questions ci-après feront l'objet de nos préoccupations ${ }^{1}$ :

- Que serait l'apport de l'exploitation minière dans la ville de Kolwezi?

- Qu'est-ce que la population attend de l'exploitation minière dans la ville de Kolwezi?

- Quel serait l'impact de l'exploitation minière sur la vie sociale dans la ville de Kolwezi?

C'est dans cette logique que nous tenterons de donner quelques éléments de réponse dans le corps du travail.

A la fin de cette rédaction, nous démontrerons quelques images d'exploitation minière qui datent de 1961 (depuis le gouvernement Moise TSHOMBE) et nous ferons l'interview à la population de Kolwezi par rapport à l'exploitation minière.

Dans la même rédaction, nous n'oublierons pas de démonter quelques impacts négatifs comme positifs.

Impact négatifs : par exemple, les éboulements dans des carrières qui font l'objet de décès, les males formations de nouveau-nés, des différentes maladies liées à la pollution des déchets jetés par certaines entreprises dans des rivières,

Impacts positifs : par exemple, la diminution de chômage, construction de routes vers l'intérieur de la ville, quelque construction d'écoles sur la ville ${ }^{2}$.

\section{INTRODUCTION}

Un Etat de Droit, soucieux de sauvegarder les droits fondamentaux de la liberté de sa population et de veiller sur la vie sociale de cette population, l'administration évite de se réserver de trop large aux entreprises qui ne remplissent pas leurs devoirs pour le bien être de la communauté.

En effet, notre étude porte sur l'impact de l'exploitation minière sur les infrastructures et la vie sociale dans la ville de Kolwezi. Il convient dès à présent, que nous définissions les concepts impacts, exploitation minière, les infrastructures ainsi que la vie sociale.

L'existence des entreprises minières dans une ville est un atout, un avantage primaire pour la population de cette entité, dès lors qu'il y a création des emplois par le gouvernement (lutte contre le chômage), contribution pour la construction de routes, amélioration des conditions de vie de la communauté, la construction des ponts, des aéroports modernes, d'une gare, les construction des écoles, des universités ou des centres des recherches, l'aménagement des jardins botaniques ou zoologiques, l'aménagement d'un trottoir et la construction d'un centre hospitalier, d'un marché public, l'aménagement des terrains des sports et des lieux de loisir, etc.

Ainsi donc, l'existence des entreprises minières dans une entité devient un atout dans la vie sociale de la population. Ces quelques exemples cités ci-haut, illustrent les cas le plus

1 J. RIVERO et J.WALINE, Droit administratif, 18e Edition, Dalloz, Paris, 2000, p 10.

2 KUYUSA et K.SHOBA, Initiation aux méthodes de recherché en Science Sociale, PUL, 1945, P 1. 
frappant est l'intérêt public qui peut se manifester et où l'administration peut valablement intervenir si les devoirs et obligations ne sont pas remplis.

Jean RWEMO et Jean WALINE renseignent que le but de l'administration, c'est la poursuite de l'intérêt public, le domaine de l'administration est constitué des nécessités auxquelles l'initiative privée ne peut répondre et sont vitales pour la communauté entière et pour chacun de ses membres.

C'est la sphère de l'intérêt public.

Le moteur de l'action administrative est la poursuite de l'intérêt général, ou encore de l'utilité publique, ou dans une perspective plus philosophique du bien commun. L'intérêt général est un ensemble de nécessités humaines. Ainsi, l'exploitation minière serait pour la communauté un intérêt général dans le sens où elle améliore les conditions de vie de l'entité.

Dans le cadre de nos recherches, la problématique désignerait l'ensemble d'idées qui signifieront la position du problème suscité par le sujet d'étude. Ainsi, en ce qui concerne le présent travail, les questions ci-après feront l'objet de nos préoccupations ${ }^{3}$ :

- Quel serait l'apport de l'exploitation minière dans la ville de Kolwezi?

- Qu'est-ce que la population attend de l'exploitation minière dans la ville de Kolwezi?

- Quel serait l'impact de l'exploitation minière sur la vie sociale dans la ville de Kolwezi? C'est dans cette logique que nous tenterons de donner quelques éléments de réponse dans le corps du travail.

A la fin de cette rédaction, nous démontrerons quelques images d'exploitation minière qui datent de 1961 (depuis le gouvernement Moise TSHOMBE) et nous ferons l'interview à la population de Kolwezi par rapport à l'exploitation minière.

Dans la même rédaction, nous n'oublierons pas de démonter quelques impacts négatifs comme positifs dans le cadre de nos recherches.

Impact négatifs : nous constatons, les éboulements dans des carrières qui font l'objet de décès, les males formations de nouveau-nés, des différentes maladies liées à la pollution des déchets jetés par certaines entreprises dans des rivières,

Impacts positifs : ici nous constatons par exemple, la diminution de chômage, construction de routes vers l'intérieur de la ville, quelques construction d'écoles sur la ville $^{4}$. sur la ville nous constatons également un équilibre social donc une circulation des monnaies sur le marché.

4 KUYUSA et K.SHOBA, Initiation aux méthodes de recherché en Science Sociale, PUL, 1945, P 1. 


\section{L'EXPLOITATION MINIERE.}

Comme l'autorise la loi, un projet minier est un projet qui se base plus sur les minerais tels que Cuivre, Nickel, Cobalt, Or, Argent, Plomb, Zinc, ....Pour accomplir ce projet, quelques phases nous sont proposées ${ }^{5}$ :

\section{a. Phase prospective}

S'agissant de cette phase, un projet minier peut commencer seulement quand l'extraction et la valeur du dépôt de minerais sont connues.

A cette phase, les investisseurs font les enquêtes, l'étude de terrain, les essais de sondage, sur les milieux d'exploitation. Signalons qu'à cette phase plusieurs notions requièrent une étude d'impacts environnementaux (EIE) séparée de la phase exploratoire d'un projet minier parce que les impacts de cette phase peuvent être profonds et, parce que les prochaines phases du projet minier peuvent ne pas s'ensuivre si l'exploitation n'arrive pas à trouver de quantités suffisantes des dépôts de minerais à haute teneur.

\section{b. Phase de développement}

Si la phase précédente demande que le dépôt de minerais puisse exister et il est important que le moteur de projet débute avec la planification du développement d'une mine.

Il est à signaler que cette étape comprend plusieurs sous-étapes notamment :

- La construction des routes d'accès afin de faciliter l'approvisionnement des matériels de travail (les équipements lourds) au site minier.

- La préparation et le déblaiement du site et à cette étape, si le site d'une mine est situé dans une zone vierge et difficile d'accéder, les investisseurs peuvent avoir besoin de commencer d'abord avec le déblaiement de terrain, ensuite la construction de campement pour loger le personnel et stocker les équipements pouvant servir à l'exploitation des minerais.

c. Phase d'exploitation minière active.

Après avoir construit les routes d'accès et préparé les zones de campement pour héberger le personnel et stocker les équipements ou matériel, on peut démarrer avec les travaux d'exploitation de minerais et ainsi, les types de travaux miniers partagent un (point) aspect commun. C'est l'extraction.

Signalons à ce niveau que la première étape dans les projets miniers propose différentes méthodes pour déplacer les minerais sur le site minier. Les minerais métalliques sont emprisonnés sous une couche de sol et de roche ordinaires.

5 http/www.Mncan.gec.c q/mm-smm/bwasi mdu/key fac-pix fat/mining 3-f.html(consulté en 2014.). 
Nous allons présenter les méthodes communes dans les paragraphes qui suivent pour l'exploitation minière.

\section{d. Exploitation à ciel ouvert}

Ici le dépôt des minerais s'étend profondément dans le sous-sol nécessitant l'enlèvement des couches qui sont superposées de terre stérile terrains et des minerais. Cette exploitation fait souvent intervenir le déplacement de la végétation minière les plus déstructurée.

Du point de vue environnemental, spécialement dans les forêts tropicales, du fait que l'exploitation à ciel ouvert est utilisée pour des minerais se trouvant à une profondeur substantielle, elle fait couramment intervenir la création d'un puits qui s'étend en dessous du niveau de la nappe phréatique.

\section{e. Exploitation des placers}

Celle-ci est utilisée lorsque le métal d'intérêt est associé aux sédiments dans un lit de cours d'eau dans une plaine d'inondation. Un processus appelé abeillage hydraulique, c'est-à-dire le bouteur des dragues ou des projets hydrauliques sont utilisés pour extraire le minerais.

Cette exploitation vise spécialement à récupérer de l'or à partir de sédiments de cours d'eau et des plaines inondables, elle se produit souvent dans un cours d'eau.

\section{f. Exploitation souterraine}

Ici, une quantité minérale de terre stérile est enlevée pour accéder au dépôt de minerais. L'accès à ce gisement de minerais se fait au moyen des tunnels ou des puits. Malgré que cette exploitation soit une méthode moins destructive de l'environnement pour accéder à découvrir par décapage direct y compris l'exploitation à ciel ouvert.

g. Réouverture des mines inactive ou abandonnée et retraitement des résidus.

Quelque projet minier consiste à remanier des piles de déchet (des résidus) des mines inactives ou abandonnées ou d'anciens tas de déchets dans les mines activesCela est généralement fait lorsque les méthodes plus efficaces d'enrichissement ont rendu économique l'extraction des métaux à partir des déchets miniers anciens. Les projets miniers qui consistent uniquement à retraiter des piles de déchets de l'exploitation à ciel ouvert et l'exploitation des placers entrainent toujours les impacts environnementaux associés à la purification (enrichissement) de métaux dans les tas de déchets.

\section{BREVE DESCRIPTION DE LA VILLE DE KOLWEZI}

Nous proposerons quelques solutions pour la bonne conservation de la terre, la bonne gestion de l'environnement et de réfection ainsi que la reconstruction des infrastructures. 


\section{DU SOCIAL}

Nous avons effectué ces recherches et produit ce document afin de décrire, d'expliquer et d'éclairer les différentes situations du mode de vie sur la ville de Kolwezi, afin que tous ceux qui voudront collaborer avec nous sur le plan professionnel et qui ne connaissent pas la ville de Kolwezi où nous sommes, aient une idée générale sur la ville, concernant ses coutumes et de ses habitants. Les solutions proposées proviennent de nos considérations personnelles face à tout ce qui se passe sur place et ailleurs dans les pays. Voyons maintenant les points forts sur lesquels le constat a été fait dans ce milieu, et ce constat va aider quiconque veut savoir la réalité sur ce qui se passe dans la ville de Kolwezi.

\section{DE LA POLITIQUE ET LE MODE DE GESTION DE LA POLITIQUE INTERNE;}

Après la guerre de l'Est de la République Démocratique Congo, notre pays est dans une autre phase. La ville de Kolwezi se trouve au sud de la République Démocratique du Congo dans la province du Katanga elle fut créée par ordonnance présidentielle depuis 1971, et elle a ses propres réalités; le premier maire de la ville nommée après la libération du pays de la domination du dictateur feu MOBUTU, celui-ci fut succédé par plusieurs maires jusqu'à avoir celui qui dirige la ville actuellement. La ville est gérée de la même manière, la population bénéficie des quelques investissements de la part du gouvernement central, et quelques réalisations des biens et services rendus par le gouvernement provincial dans les différents domaines ou secteurs. La Ville de Kolwezi est constituée de deux Communes dont MANIKA et DILALA, gérées par les Bourgmestres des Communes.

\section{SITUATION GENERALE ET PARTICULIERE DU SOUS-SOL.}

La ville de Kolwezi est une entité où il y a diverses sortes de pierres précieuses sont dans son sol et sous-sol. La ville est née de l'exploitation des minerais dont les mines à ciel ouvert et des mines souterraines; exploitées par la Gécamines dans le temps et aujourd'hui avec les phénomènes boum Mining la ville de Kolwezi est envahie par plusieurs entreprises minières; ensuite elle est reconnue comme étant le poumon économique du Congo au niveau national. Cette exploitation était l'œuvre de la colonisation Belge, aujourd'hui l'exploitation se fait d'une manière artisanale; les minerais sont creusés non pas seulement par des machines, mais aussi par des hommes à la main.

\section{SITUATION GENERALE DES INFRASTRUCTURES}

Si nous avons parlé de routes dans la ville de Kolwezi, c'est parce que les autorités politicoadministratives tiennent compte de la valeur des routes pour le développement national et social pour le bien être de la communauté, mais il y a manque de volonté politique pour engager des moyens concernant la réfection des routes d'intérêt général. Le service de voirie qui existait à l'époque coloniale pour la réparation et l'entretien des routes n'existe plus. 
Certaines routes dans cette ville connaissent d'éboulements, de nid des poules, ce qui veut dire qu'après une certaine pluie, les canalisations bouchées ne permettent pas à l'eau de passer facilement, certaines parties de ces canalisations qui sont bouchées bloque le passage libre de l'eau et cette dernière par la pression déborde sur les routes et crée des érosions ${ }^{6}$. C'est une forme de carrière ouverte aux environs des maisons d'habitation qui s'écroulent souvent à cause des eaux de pluie provenant de la route non entretenue, les dégâts restent toujours, le service de voirie n'a même pas de plan urbanistique pour savoir làoù passent les canaux d'évacuation des eaux et de fosses septiques etc.

\section{L'APPORT DE L'EXPLOITATION MINIERE SUR LA VILLE DE KOLWEZI}

\section{LES ROUTES}

Les routes sont des voies de communication par excellence dans une entité donnée. En effet, tout Etat qui aspire au développement se priorise à améliorer ses voies de communication entre autre les routes. Elles permettent, le déplacement des biens et des personnes d'une entité à une autre, elles relient les différents centres, elles permettent d'approvisionner les centres urbains en d'entrées alimentaires provenant des milieux ruraux. Actuellement, les habitants des milieux ruraux produisent beaucoup de produits agricoles qu'ils ne savent pas écouler à cause de mauvais états des routes, malgré quelques efforts fournis par les autorités politico administratives au niveau local.

Kolwezi est une ville ${ }^{7}$ à vocation minière où il y a la présence de plusieurs sociétés minières. La vente des produits miniers ne se fait pas sur place, les sociétés acheminent ces produits miniers vers d'autres pays; la voie la plus favorable pour faire déplacer les produits c'est le chemin de fer. Vu l'état actuel des chemins de fer (Kolwezi-Lubumbashi), chaque jour, les produits quittent notre pays ou de la ville de Kolwezi vers d'autres pays en empruntant les routes. Vu l'état de ces dernières, ce trafique ne permet pas de les maintenir dans un bon état durant une longue période étant donné que les minerais sont transportés dans des gros camions poids lourds.

Le revenu que produit cette exploitation minière est énormément capable d'aider notre gouvernement à améliorer l'état des routes en les réhabilitant pourquoi pas en construire des nouvelles.

Les sociétés minières disent avoir contribuées chaque année au trésor public une somme considérable. Est-il possible que la contribution soit considérable alors que les routes continuent à se délabrer et s'affaisser d'avantage? Cela nous pousse à dire qu'il y a manque de volonté dans le chef du gouvernement tant national que local de pouvoir songer au bien être de la communauté.

6 Interview sur la population habitant du Quartier Latin de la Commune de Manika/Kolwezi.

7 www.rdc.kolwezi.com, site publie par l'éditeur TSHIKWATA SAM, Kolwezi, 2007, consulté le 17 février 2014. 
A part la route nationale qui va de Kolwezi à Lubumbashi et laquelle a été aménagée, il $\mathrm{y}$ a d'autres routes qui mènent vers les milieux ruraux que le gouvernement n'a jamais songé à réaménager pour permettre l'évacuation des produits agricoles vers la ville de Kolwezi. Ces routes sont parfois fréquentées par certains véhicules appartenant aux entreprises minières pour leur besoin d'exploitation (recherche des minerais en même temps l'exploitation). C'est le cas des routes tel que : Kolwezi vers Kisanfu., Kolwezi vers Kamowa, Kolwezi vers Kawama etc.

L'aménagement de ces routes permettra à la population de ces villages d'acheminer leurs produits vers le centre-ville; et cela permettra à la population de s'épanouir avec le moyen financier qu'elle aura et facilitera le transport parce que les routes sont bonnes. Il y aura également changement de mode de vie, de mentalité, culture et les villages pourront se développer petit à petit, bref l'objectif poursuivi c'est le désenclavement des milieux ruraux. La route la plus courte partant de Kolwezi vers la Zambie (Solwezi) est dans l'oubliette et pourtant c'est la route la plus courte et favorable.

\section{LE CHEMIN DE FER}

Le chemin de fer est le moyen le plus ancien qui facilite le transport des biens et des personnes de Lubumbashi vers Kolwezi jusqu' à Dilolo et vice versa. Depuis que la société de chemin de fer a connu une chute, il y a beaucoup de difficultés pour le transport des biens et des personnes sur ces tronçons.

Les produits miniers sont transportés par de longs camions remorque au profit de certaines personnes et pourtant la société nationale de chemin de fer qui est une entreprise publique reste en souffrance et ne profite à rien. Or, si le chemin de fer est réhabilité, cela profitera à tous, c'est-à-dire la population aura la faciliter de se déplacer et d'acheminer leurs produits vers le centre. Les entreprises minières qui transportent les produits miniers par voie routière se verront avantageux, en de quantité, en empruntant le chemin de fer ${ }^{8}$.

Ainsi, pour permettre de résoudre ces problèmes, il appartient aux entreprises minières qui tirent profits dans le secteur minier dont le centre est la ville de Kolwezi de songer à l'amélioration des routes et chemins de fer par leur contribution remarquable, mais du moins nous saluons ici le gouvernement central de par sa diplomatie il a inauguré le port de LOBITO, depuis le mois de janvier 2015 frontière qui relie la République Démocratique du Congo et l'Angola ce maintenant que l'on constate petit à petit un arrangement effective de la route et le chemin de fer reliant Angola et R D Congo.

8 Interview aux agents de la Société Nationale de Chemin de fer du Congo/Kolwezi, 2014. 
DE LA CONTRIBUTION DE L'EXPLOITATION MINIERE SUR LA VIE SOCIALE A KOLWEZI.

\section{LA CREATION D’EMPLOI}

La constitution prévoit que tout congolais a droit à un emploi décent, que l'emploi est un droit, tout congolais a le devoir de contribuer au développement du pays. Les investisseurs qui viennent créer des entreprises à Kolwezi devraient s'inscrire dans cette logique. Il est à noter que la plupart des investisseurs amènent leurs mains d'œuvres qui parfois coutent chère, alors que sur place à Kolwezi, se trouve une main d'œuvre moins couteuse ${ }^{9}$.

Le code du travail demande aux employeurs de privilégier d'abord les congolais, ne recourir aux expatriées que, quand en RDC en général ou à Kolwezi en particulier, il y a un manque ou une insuffisance d'une main d'œuvre qualifiée.

Il est à signaler qu'un effort a été fourni par des investisseurs déjà installés mais la main d'œuvre locale est encore abondante.

\section{LA REMUNERATION}

Tout travail mérite un salaire, la rémunération est l'ensemble d'avantages accordés au salarié en rapport avec la qualité des services qu'il offre à l'employeur que le simple salaire de base. La rémunération d'un travail à la tache ou aux pièces doit être calculer de telle sorte qu'elle procure au travailleur des capacités moyennes et travaillant normalement, un salaire au moins égale à celui du travailleur rémunéré autant et effectuant un travail analogue. La rémunération est fixée par des contrats individuels conclus entre travailleur et employeur ou par voie des conventions collectives. Rémunération doit être stipulée en monnaie ayant cours légal en République Démocratique du Congo. Son montant est déterminé soit à l'heure, soit à la journée, soit à la semaine ou au mois, soit à la pièce, soit à la tâche ${ }^{10}$.

L'employeur est tenu d'accorder une rémunération qui tient compte des conditions de vie, afin de lui permettre de nouer les deux bouts du mois et de subvenir aux besoins croissants de ses salariés ${ }^{11}$.

L'on apprend une bonne politique en matière de rémunération dans certaines entreprises, vraies dans certaines autres un effort doit continuer à être fourni.

9 Art 36 de Constitution de République Démocratique du Congo, du18 février 2006 tel que modifié le 20 janvier $20011 \mathrm{p} 15$.

10 Code du travail, loi N0 015/2002, du 16/10/2002, p. 28-29.

11 Cfr note 20 op.cit. p. 31-32. 


\section{INFRASTRUCTURES EDUCATIVES}

Les écoles sont des centres par excellence pour la formation des élites capables de concevoir, planifier, inventer, diriger, c'est dans les écoles qu'on forme les hommes dont les sociétés minières ont besoin pour l'amélioration des leurs activités.

Nous constatons actuellement que des entreprises minières qui s'implantent actuellement importent la main d'œuvre étrangère faute d'une main d'œuvre locale qualifiée dans divers domaines.

Lorsqu'une société minière s'installe, elle doit créer des écoles pour former des divers cadres de mérite dont elles auront besoin pour son entreprise. Dans notre code minier, il est prévu que chaque entreprise investisse dans le social, en créant des écoles, des hôpitaux, des routes, des champs ${ }^{12}$.

\section{INFRASTRUCTURES SANITAIRES}

Les hôpitaux sont des services spécialisés pour assurer la bonne santé des personnes. Le code du travail oblige les employeurs à avoir des structures des soins propres à leurs entreprises pour soigner leurs salariés malades ou qui connaissent des accidents de travail. Mais également pour répondre à l'exigence du code minier qui veut que chaque entreprise ait dans son programme le projet d'investissement dans le social de la population congolaise. Quand la masse ouvrière est malade ou non soignée, il est difficile à ces dernières de fournir une bonne prestation à l'entreprise, à Kolwezi il n y a que les entreprise MUMI, K.C.C, et Gécamines qui ont leurs hôpitaux et les autres n'en ont pas mais sont par contre abonnées dans certains centres de santé privés.

\section{SECURITE ALIMENTAIRE.}

Compte tenu de l'écartement du milieu urbain et du marché, certaines sociétés distribuaient des produits ouvriers aux travailleurs pour leur permettre de les enquérir sur place. C'est le cas de la Gécamines et de la SNCC à l'époque où ces sociétés fonctionnaient convenablement, mais à l'heure actuelle ce système a disparu et aucune sociétéminière ne l'applique. Or, si ces sociétés le font, les travailleurs qui font parties de la population vont bénéficier de ces produits et cela aura un impact sur la vie sociale ou la fréquentation de chaque jour sur marché diminuera.

\section{L'AGRICULTURE.}

L'agriculture est un secteur le plus important dans la société. Les produits agricoles sont reconnus comme aliment de base dans chaque milieu social. Or, pour en avoir il faut culti-

12 Constat fait sur la ville de Kolwezi (Notre propre constat après analyse et recherche dans la période allant du mois de Février à Juin 2014. 
ver. Dans un pays où l'agriculture est négligée, il y a toujours une crise alimentaire, d'où il faut encourager les agriculteurs en créant un système d'exonération de certaines taxes concernant les intrants agricoles à l'entrée, même les instruments servant à l'agriculture.

La bonne politique que peut être entreprise par les autorités politico-administratives est celle d'exiger à chaque société minière de toute catégorie d'avoir une concession dans laquelle elle doit cultiver les produits de premier nécessité tels que le maïs, le haricot, l'arachide, le manioc etc., et qu'à la fin de chaque année, elle fait le rapport au service de l'agriculture. Un autre système qui aurait existé autrefois, est celui dont nous donnons l'exemple de la société SODIMIZA de l'époque. Celle-ci encourageait ses travailleurs à avoir des champs en leurs donnant les intrants agricoles et même les outils de travail et mettre à leur disposition un tracteur. A la fin et lors de la récolte, ces travailleurs donnaient à la société un petit nombre de sacs de leur récolte et cela permettait à la société de récupérer ses dépenses sans intérêts.

Pour éviter les absences afin de promouvoir la production au sein de l'entreprise, il est souhaitable que l'entreprise ait dans sa politique de gestion, un champ de maïs question de subvenir aux besoins alimentaires des travailleurs.

Ce système peut être aussi appliqué par les sociétés minières actuelles en vue de lutter contre les crises alimentaires à Kolwezi.

\section{DES ATTRIBUTIONS DE DIFFERENTS ORGANES EN MATIERES DES MINES}

\section{LE PRESIDENT DE LA REPUBLIQUE}

Dans le cadre du nouveau code minier, les attributions du Président de la République sont nettement précisées $^{13}$.

En effet, outre sa compétence relative à la promulgation du règlement minier pour l'exécution du code, le chef de l'Etat est compètent pour classer les substances minérales en produits de carrières et inversement, il a également le pouvoir de déclarer certaines substances «substances réservées », il confirme la réservation faite par le ministère des mines en cas de gisement à soumettre à l'appel d'offres.

\section{LE MINISTERE DES MINES}

Pour le ministère des mines ses attributions traditionnelles, tels que l'octroi des droits miniers, l'établissement des zones d'exploitation artisanales et l'agrément des comptoirs d'achats, l'octroi des droits de carrières pour les substances minérales et autres matériaux de constructions à usage courant, ${ }^{14}$ la réservation des gisements à soumettre à l'appel d'offres l'approbation des hypothèques minières, l'agrément des mandataires en mines et 
carrières, la délivrance des autorisations de transformation des produits d'exploitation des minerais à l'état brut.

\section{LE GOUVERNEUR DE PROVINCE}

Pour le Gouverneur de Province et le chef de division provinciale des mines; le premier intervient comme autorité compétente dans l'octroi des cartes de négociant des produits d'exploitation artisanale, l'ouverture des carrières pour les travaux d'utilité publique sur les terrains tandis que le second est compétent pour l'octroi des cartes de creuseur, des droits d'exploitation des carrières pour les matériaux de constructions à usage courant ${ }^{15}$.

\section{LE CADASTRE MINIER}

S'agissant du cadastre minier dont les attributions est chargé d'administration, chargé de la dotation, de la personnalité juridique et de l'autonomie financière afin de lui permettre de percevoir et de gérer à son profit les faits de dépôt des dossiers et les droits de superficialité annuel par carré. Elle contribue une qualité à d'autres organes qui interviennent dans l'administration du code minier. Le cadastre minier relève de la tutelle des ministères des mines et des finances. ${ }^{16}$

\section{DIRECTION GEOLOGIQUE ET DES MINES}

S'agissant de la Direction Géologique et de ceux de la direction des mines, ne gère plus la procédure d'octroi de la déchéance ou d'annulation des droits miniers et de carrières, la direction de géologie n'intervient pas non plus dans les procédures mais elle se concentrera sur les études géologiques à grandes échelles ${ }^{17}$.

\section{LA CONTRAINTE D'ORDRE ENVIRONNEMENTAL}

Au regard des contraintes d'ordre environnemental, le nouveau code a prévu des dispositions en vue de veiller efficacement au travers du service chargé de la protection de l'environnement minier ${ }^{18}$, ce service intervient dans l'instruction technique du plan d'atténuation et de réhabilitation de l'environnement en abrégé P.A.R dans l'étude d'impact environnementale du projet minier en sigle P.G.E.P.

Il est également à préciser qu'en dehors du ministère des mines, des services et des organes chargés de l'administration du code minier, aucun autre service ou institution pu-

15 Article 11, LOI N 007/2002 DU 11 JUILLET 2002 PORTANT CODE MINIER, p. 42.

16 Article 13, LOI N ${ }^{\circ}$ 007/2002 DU 11 JUILLET 2002 PORTANT CODE MINIER,p.42.

17 Article 13,LOI N 007/2002 DU 11 JUILLET 2002 PORTANT CODE MINIER, p. 44.

18 Nouveau Code minier, Article 15, p. 44. 
blique ou étatique n'est compétent pour appliquer les dispositions du code minier et ses mesures d'exécution.

\section{DE LA BONNE GOUVERNANCE DANS LE SECTEUR MINIER}

Pour une bonne gestion des mines dans le monde des affaires afin d'arriver à un développement durable comme objectif, il a été permis au premier ministre Britannique TONY BLAIR, lors du sommet mondial sur le développement durable en 2002 à JOHANNESBOURG, principalement en Afrique du Sud; il a été créé un Organisme International dénommé Initiative pour la Transparence des Industries Extractives en sigle « ITIE » ${ }^{19}$.

L'ITIE, est un processus à travers lequel les parties prenantes mettent à la disposition des populations à travers la rédaction des rapports périodiques par des consultants indépendants, les revenus et les recettes de l'Etat issus de l'exploitation de signature, les bonus de production, les égalités les dividendes et les taxes diverses.

A leur avis : « la bonne gouvernance dans le secteur minier passe inévitablement par la mise en place effective de l'ITIE qui doit également être opérationnelle dans les provinces, en vue de renforcer le système de traçabilité des produits miniers et de recettes ainsi générées, de certifier les méthodes de production et de combattre les exportations frauduleuses.

Ils ont aussi fait remarquer que : « le progrès enregistré dans le secteur minier ne peut plus continuer à être géré par une administration publique obsolète, dont les structures aussi bien nationales que provinciales n'ont pas connu de reformes depuis la mise en vigueur du nouveau Code minier».

« Contrairement au Code foncier, le Code miner n'a reconnu aux autorités provinciales et locales que très peu de pouvoir dans la procédure d'octroi et de retrait des concessions minières aussi bien que dans la gestion et la surveillance environnementale. Les concessions minières sont parfois attribuées par les autorités nationales sans tenir compte de certaines réalités sur le terrain », se sont encore plaint des gouverneurs de province.

" Grandes » recommandations. »

«Parmi les grandes recommandations » de la conférence de Lubumbashi, le Premier ministre a épinglé la révision du Code minier, la transformation locale des produits miniers avant leur exportation, la mise en place d'un service géologique national, un contrat de gouvernance, une meilleure traçabilité des recettes minières. Plus probablement, les recommandations ont porté sur le cadre légal et règlementaire ainsi que sur le cadre institutionnel.

Dans le premier cas, la conférence de Lubumbashi a recommandé de « finaliser la révision du Code minier avec toutes les parties prenantes, régler la problématique de l'optimisation de la fiscalité minière dans le cadre de la révision du Code minier, harmoniser les textes légaux, maintenir le processus des publications des contrats, réviser le plan directeur d'aménagement du territoire en intégrant tous les domaines sectoriels en rapport avec l'in- 
dustrie extractive, finaliser l'élaboration des mesures d'application de la loi portant principes fondamentaux relatifs à la protection de l'environnement et la publier $»^{20}$.

En ce qui concerne le cadre institutionnel, la conférence de Lubumbashi a recommandé d' « harmoniser les rapports entre institutions et services en vue de régler les problèmes de conflit de compétence, d'adapter le Code minier aux aspects de la décentralisation, de renforcer les capacités des institutions, de finaliser la réforme des entreprises publiques, de créer et borner les nouvelles zones d'exploitation artisanale (ZEA), d'harmoniser les limites des aires protégées entre le CAMI et l'ICCN, répertorier tous les périmètres miniers qui chevauchent avec les aires protégées ».

S'agissant des opérateurs miniers, il leur est recommandé notamment de : « respecter la délimitation des zones d'exploitation artisanale, d'assurer le transfert d'expertise, des compétences et des technologies, de collaboration avec les coopératives minières du ressort des activités minières concernées $»$.

Nous reconnaissons que la transparence financière est un moyen susceptible de contribuer à l'amélioration du climat pour l'investissement direct intérieur et étranger. Nous croyons en principe et à la pratique de la responsabilité du gouvernement devant tous les citoyens en ce qui concerne l'intendance de flux de recettes et de dépenses publics. Nous nous engageons à encourager le respect de haut niveau de transparence et de responsabilité dans la vie publique, le fonctionnement de l'Etat et le monde du commerce.

Lorsqu'il s'agit de trouver des solutions, nous considérons que toutes les parties prenantes, à savoir le gouvernement et leur agence, les entreprises extractives, les sociétés de service, les organisations multilatérales, l'organisation financière, les investisseurs, et l'organisation non gouvernementale, ont des contributions pertinentes à apporter.

\section{LES CRITERES DE L'ITIE}

Le paiement matériel versé par les entreprises au gouvernement au titre de l'exploitation pétrolière, gazière et minière et toutes les recettes matérielles reçues par le gouvernement de la part des entreprises pétrolières, gazières et minières est publié et diffusé régulièrement au grand public sous une forme accessible, complète et compréhensible.

Le gouvernement d'accueil élabore un plan de travail public financièrement viable relatif aux éléments ci-dessus avec le concourt des institutions financières internationales, le cas échéant, ce plan étant assorti des cibles mesurables d'un calendrier de mise en œuvre et d'une évaluation des contraintes éventuelles sur le plan de capacité.

La société civile participe activement à la con conception, au suivi et à l'évaluation à ce processus et apporte sa contribution au débat public ${ }^{21}$.

21 4e Rapport ITIE/RDC, Exercice 2011. 


\section{L'EFFECTIVITE DE L'ITIE}

Les participants à la conférence ont examinée durant deux jours sur toutes les facettes les problèmes de la gouvernance du secteur minier caractérisée fondamentalement par la corruption et la fraude alors qu'il doit contribuer à la croissance économique du pays.

Ils ont noté que le sol et le sous-sol congolais regorge des substances minérales connues dont une dizaine et réellement mise en valeur, dont le diamant, le cuivre, le cobalt, l'or, le manganèse, le zinc, la cassitérite, le colombo-tantalite (coltan), le wolframite, le niobium, le germanium.

Ces potentialités minières doivent cependant être exploitées en vue de leur transformation en richesse pour le développement économique et social du pays, dans ce contexte, force est de constater que le secteur minier de la RDC est confronté à plusieurs défis notamment : l'exploitation illégale et le trafic illicite transfrontalier des minerai, l'évasion fiscale, la criminalisation dans ce secteur à cause des conflits armées récurrents dans les zones miniers, la baisse de recette d'exportation, la corruption et la fraude.

Ils en ont conclu que ces difficultés affectent la transparence et la gouvernance du secteur prive le gouvernement des moyens importants pour la réalisation du bienêtre social.

Face à tous ces problèmes, le Président de la République Démocratique du Congo, a alors assigné au gouvernement la mission d'instaurer les meilleures pratiques de gestion notamment par l'adhésion en 2005 de la RDC à l'initiative pour la transparence dans les industries extractive (ITIE et à l'HOADA).

Pour que la bonne gouvernance mène à la croissance, il a souvent était évoqué trois principaux facteurs. Il s'agit de la maitrise des couts d'exploitation et de la culture de la rente des cours internationaux des produits de base dont la fixation des prix ne dépendent pas forcement de la volonté des pays producteurs de la stabilité politique qui est un préalable pour attirer des capitaux importants des investisseurs locaux et étrangers ont rappeler les gouverneurs des provinces dans un message lit par leur porte-parole.

Deux facteurs en particulier distinguent l'ITIE des autres efforts pour partager plus largement le bénéfice des industries extractives.

- Premièrement elle met l'accent explicitement sur la transparence qui aide à créer une culture de responsabilité publique et à gagner la confiance public dans les industries extractives.

- Deuxièmement, facteur qui rend l'ITE unique est sa structure à prise des participations multi actionnaire, c'est-à-dire elle implique le gouvernement, les entreprise et la société civile; des dirigeants des trois secteurs soutiennent l'initiative au niveau international et des principes et critères pour les programmes ont été développés au vue des expériences à travers le monde. 


\section{BUT DE L'ITIE}

Le but l'ITIE est de renforcer de bonne gouvernance en améliorant la transparence et la responsabilité (obligation de rendre compte) dans le secteur des industries extractives à travers la collecte, la vérification, la conciliation et la publication de tous les paiements effectués par les Entreprises publiques et privées au compte de l'Etat.

Deux facteurs en particulier distinguent l'ITIE des autres efforts pour partager plus largement les bénéfices des industries extractives, premièrement, elle met l'accent explicitement sur la transparence, qui aide à créer une culture de responsabilité publique et à gagner la confiance publique dans les industries extractives.

\section{AVANTAGE DE L'ITIE ${ }^{22}$}

\section{a. Pour les gouvernements :}

Une bonne gestion des recettes provenant des ressources permet d'améliorer la gestion de ces ressources et devrait favoriser une grande stabilité économique et politique. Ceci peut à son tour aider à empêcher les conflits qui touchent les secteurs pétroliers, miniers et gaziers. La mise en œuvre permet également d'améliorer le climat d'investissement parce qu'elle indique clairement aux investisseurs et aux institutions financières internationales que le gouvernement s'est engagé en faveur d'une grande transparence; ainsi les gouvernements qui mettent en œuvre l'initiative pour la transparence de l'industrie extractive bénéficie de la mise en œuvre d'une procédure standardisée et reconnue.

\section{b. Pour les entreprises et les investisseurs :}

L'instabilité politique que provoque une gouvernance opaque constitue évidemment une menace pour les investissements étant en forte intensité des capitaux et dépendant de la stabilité à long terme pour produire un rendement. La réduction d'une telle instabilité est avantageuse; la transparence peut également contribuer à créer une situation équitable pour toutes les entreprise et en rendant public ce qu'elle verse au gouvernement, une entreprise illustre la contribution que son investissement apporte au pays.

\section{c. Pour la société civile}

Les avantages pour la société civile tiennent du volume plus important des informations disponibles dans le domaine public sur les recettes que le gouvernement gère au nom des citoyens; ces derniers pouvant ainsi plus facilement demandée des comptes à leur gouvernement et faire en sorte à travers plusieurs autres initiatives et des plaidoyers que les re- 
cettes soient effectivement utilisées pour des investissements nécessaires au profit de la population et pour le développement durable de la nation.

Les organisations venant de la société civile jouent un rôle clé dans l'initiative pour la transparence des industries extractives, y compris pour préconiser l'engagement, surveiller les processus de mise en œuvre et aider à garantir que le rapport de l'ITIE soit largement compris.

\section{CRITIQUES ET SUGGESTIONS}

\section{CRITIQUES}

Avant la mise en application du nouveau code minier, la Générale des Carrières et des Mines disposait dans la province du Katanga uniquement des concessions pour des gisements de cuivre, cobalt, et de Zinc sur environ $18.900 \mathrm{~km}^{2}$ pour les Cuivres et associés à d'autres minerais de $1400 \mathrm{~km}^{2}$ pour l'Etain et autres accompagnateurs, mais toutes ces réalisations avaient comme objectif, le développement de la population dans tous les secteurs, mais seulement avec une administration faite de qualité qui a fait que la Gécamines a réalisé autant de projets, et surtout que la Ville de Kolwezi est à vocation minière, c'est avec raison que la Gécamines eut à fournir beaucoup d'effort pour l'épanouissement de la ville, la rendant plus forte, efficace auprès des autres Ville de la République Démocratique du Congo parce que de tout ce temps Kolwezi c'est un District.

Mais tout est parti de la bonne ou mauvaise politique des dirigeants; surtout avec la philosophie selon la création ou la venue du code minier qui modifié les principaux minerais extraits à savoir : le cuivre, cobalts, zinc de Nickel et accessoirement d'uranium en partie illégalement exploité par des enfants, car il faut protéger la vie humaine surtout dans l'artisanal. Car l'artisanal a comme souci majeur de créer une « classe moyenne », mais d'autres structures ont été créée pour cette fin.

Mais plus loin, l'on a constaté par après que la politiques a abandonné sa mission primordiale pour s'intégrer totalement aux mines depuis les années à nos jours, les opérateurs politiques ont fait des mines une affaire politique. C'est-à-dire un opérateur politique congolais à tout le niveau qu'il soit, il est en même temps actionnaire, sous-traitant, ou entrepreneur voire même patron direct d'une société minière soit encore ses enfants, neveux; donc, c'est un système déjà créé.

Il existe à Kolwezi plusieurs sociétés et entreprises minières. Avec les représentations à la province et à la capitale de la République mais quelque part il y a toujours la présence d'un homme politique qui agit directement comme indirectement à la tête d'une société minière. Avec un tel agissement, comment faire appliquer la loi dans le domaine minier, où lorsqu'on crée le cadre minier, le premier à profiter c'est abord l'opérateur politique en commençant par les membres du cadastre miniers eux-mêmes. C'est difficile et compliqué. 


\section{SUGGESTIONS}

Il est possible d'interdire, sanctionner, arrêter, blâmer, suspendre, limoger, quiconque operateur politique qui se permettrait de se retrouver en même temps operateur minier quelque part; qu'il opère un choix entre les deux, soit c'est la politique soit les affaires.

Ensuite, qu'on interpelle à toutes les sociétés minières, d'embaucher les nationaux ou autochtones et avoir un emploi permanent, dont un " mot » d'exiger aux entreprises minières de copier ou d'emboiter les pas selon le système politique du travail Gécamines, nous pensons à ce moment-là, les sociétés et entreprises minières auront un impact sur la ville de Kolwezi pas seulement sur le plan infrastructurel, social, mais aussi environnemental, culturel, et autres innovations; ensuite libérer les mines aux politiciens, que les mines ne soient le cheval de bataille des hommes politiques comme c'est le cas dans notre pays la R.D. Congo.

Et surtout de créer un organe permanent provincial soit national qui est indépendant avec comme mission de coordonner, de contrôler, faire rapport à qui de droit, c'est à dire qu'on crée une institution indépendante provinciale chargée de contrôler et faire le suivi concernant les opérations, minières comme c'est le cas avec la direction de recette du Katanga En sigle « DRKAT » mais cette fois-là que ça soit de même sur les mines.

Le coordonnateur va chapeauter ou aura la main mise sur tous les mining qui existent au niveau provincial selon la gestion soit aussi national avec comme mandat, faire appliquer les système de la politique et de l'administration du travail Gécamines, à notre niveau nous pensons que tout ce que nous avions relevé dans les infrastructures et social vont se produire peut être encore dans la ville de Kolwezi et partout où il y a les mines au cas contraire ça sera le péril en demeure, donc plus pire que ce que nous vivons actuellement.

\section{CONCLUSION}

En guise de conclusion, pour nous, la ville de Kolwezi est à vocation minière, mais les minerais sont des ressources épuisables. Alors il est souhaitable d'envisager un autre programme d'investissement, c'est-à-dire ce que les mines produisent soient remplacé par les engins pour l'agriculture a grand échelle ou l'Etat au niveau local peut déjà amorcer car l'espace pour l'agriculture, l'élevage et autres car l'espace est très grand sur toute l'étendue de la ville de Kolwezi.

L'Etat au niveau local a tout intérêt d'en profiter le temps car à Kolwezi il y a trop de discours, trop des théories qui ne servent absolument à rien. Trop de forum de renforcement de capacité après mines, trop de plaidoyer mais sur terrain rien ne se gagne. que donc, une interpellation aux opérateurs politiques et à tout le niveau d'avoir une prise de conscience pour l'avenir de la ville de Kolwezi qui court des risques de disparaitre sur la carte politique et administrative de la R.D.Congo à cause de l'envahissement des expatriés en complicité avec les hommes politiques congolais pour l'exploitation des minerais qui font la fierté de la ville. 
Donc, à ce jour, accuser seulement les expatriés, c'est un faux débat car dit-on : l'ennemie du Congo c'est le congolais lui-même et non le visiteur ou l'étranger. Donc nous devons incarner et protéger nos valeurs patrimoniales et culturelles sans accuser les innocents. Car ils font tout avec notre contribution et engagement ferme.

Parce qu'à Kolwezi l'exploitation minière est devenue un casse-tête plus qu'un fléau, la matérialisation ou l'existence de la ville de Kolwezi c'est grâce à l'existante de la Gécamines sans laquelle Kolwezi serait un village semblable au territoire donc Lhomme politique a tout intérêt de revoir sa politique pour l'intérêt de bien-être de tous les congolais en général et de Kolweziens en particuliers.

\section{BIBLIOGRAPHIE}

\section{TEXTES DES LOIS}

La constitution de la République Démocratique du Congo, du18 février 2006 révisée Nouveau Code minier, du 15 juillet 2002

Code du travail, du 16 octobre 2002

Rapport ITE/RDC, Exercice 2011 - 2014

\section{OUVRAGES}

J. RIVERO et J.WALINE, Droit administratif, Dalloz, Paris, $18^{\mathrm{e}}$ Ed., 2000

KUYUSA ET K. SHOBA, Initiation aux méthodes de recherche en Sciences Sociale, P.U.L, 1945.

\section{SITE INTERNET}

http/www.rdc.kolwezi.com

http/www.Mncan.gec.c q/mm-smm/bwasi mdu/key fac-pix fat/mining 3-f.html 